\title{
National Trends in Pediatric Systemic Lupus Erythematosus Hospitalization in the United States: 2000-2009
}

\author{
Andrea M. Knight, Pamela F. Weiss, Knashawn H. Morales, and Ron Keren
}

\begin{abstract}
Objective. In the setting of recent healthcare advances and emphasis on reduced spending, we aimed to characterize US trends in inpatient healthcare use and mortality for pediatric systemic lupus erythematosus (SLE).

Methods. We performed a retrospective, serial, cross-sectional analysis of the national Kids' Inpatient Database (for 2000, 2003, 2006, and 2009). We identified patients with SLE aged 2 to 21 years using an International Classification of Diseases, 9th revision (ICD-9) code of 710.0 listed as a discharge diagnosis. Using sampling weights, we estimated trends in hospitalization, inpatient mortality, procedure rates, and length of stay (LOS). We analyzed patient and hospital-specific risk factors for mortality and LOS, and compared those outcomes to those without SLE.

Results. We identified 26,903 estimated pediatric SLE hospitalizations. The hospitalization rate of 8.6 (95\% CI 7.6-9.6) per 100,000 population and mean LOS of 5.9 days (95\% CI 5.6-6.2) were stable over time. We found a significant downward trend in mortality, decreasing from $1 \%$ to $0.6 \%$ $(\mathrm{p}=0.04)$, which paralleled a less pronounced trend for those without SLE. The rate of dialysis, blood transfusions, and vascular catheterization procedures increased. Patients with SLE nephritis and non-white race were at risk for increased healthcare use and death.

Conclusion. Pediatric SLE hospitalization rate and LOS remained stable, but inpatient mortality decreased as the rate of common therapeutic procedures increased. More research is needed to understand the drivers of these relationships. (First Release Feb 1 2014; J Rheumatol 2014;41:539-46; doi:10.3899/jrheum.130592)
\end{abstract}

Key Indexing Terms:

SYSTEMIC LUPUS ERYTHEMATOSUS MORTALITY

Systemic lupus erythematosus (SLE) is a chronic autoimmune condition with potential for significant morbidity and mortality, thereby requiring frequent

From the Division of Rheumatology, the Division of General Pediatrics, and the Center for Pediatric Clinical Effectiveness, Children's Hospital of Philadelphia; the Center for Clinical Epidemiology and Biostatistics, University of Pennsylvania, Philadelphia, Pennsylvania, USA.

Supported by US National Institutes of Health grant 5T32HD060550-03 (to Dr. Knight).

A.M. Knight, MD, Assistant Professor of Pediatrics, University of Pennsylvania, Perelman School of Medicine, Division of Rheumatology, and Center for Pediatric Clinical Effectiveness, Children's Hospital of Philadelphia; P.F. Weiss, MD, MSCE, Assistant Professor of Pediatrics, University of Pennsylvania, Perelman School of Medicine, Division of Rheumatology, Children's Hospital of Philadelphia, and Center for Pediatric Clinical Effectiveness; K.H. Morales, ScD, Assistant Professor of Biostatistics, University of Pennsylvania, Perelman School of Medicine, Center for Clinical Epidemiology and Biostatistics, University of Pennsylvania; R. Keren, MD, MPH, Professor of Pediatrics, University of Pennsylvania, Perelman School of Medicine, Center for Clinical Epidemiology and Biostatistics, University of Pennsylvania, Division of General Pediatrics and Center for Pediatric Clinical Effectiveness, Children's Hospital of Philadelphia.

Address correspondence to Dr. A.M. Knight, MD, Division of Rheumatology, Children's Hospital of Philadelphia, 3501 Civic Center Blvd., Philadelphia, Pennsylvania 19104, USA.

E-mail: knightan@email.chop.edu

Accepted for publication November 25, 2013
PEDIATRIC

\section{HOSPITALIZATION} LENGTH OF STAY

healthcare contact for optimal management and outcomes. Pediatric-onset SLE represents $15-20 \%$ of overall SLE cases, and is associated with an increased risk for aggressive clinical course and major organ damage in comparison to adults ${ }^{1}$. Pediatric SLE hospitalizations are significant events, indicating more severe disease, disrupting psychosocial and school functioning, and contributing to high healthcare use. Further understanding of the factors contributing to hospitalization in pediatric SLE may therefore improve clinical outcomes, quality of life, and healthcare use for these patients.

Estimates of healthcare use and mortality for adults with SLE are established in the literature. Several studies of adults with SLE in the United States have found high resource use in these patients ${ }^{2,3}$, and a study of US hospitalizations for adult SLE found that 1 in 30 hospitalizations resulted in death ${ }^{4}$. A few studies also describe increased healthcare use for children with SLE ${ }^{5,6,7}$; however, the relationship between resource use and outcomes such as mortality remains unclear. Importantly, these variables may also be changing over time, given recent advances in SLE treatment such as the recommendations for use of mycophenolate mofetil (MMF) therapy ${ }^{8,9}$, other regimens for

Personal non-commercial use only. The Journal of Rheumatology Copyright @ 2014 . All rights reserved. 
reduced exposure to cyclophosphamide toxicity ${ }^{8,9,10,11}$, and the introduction of $\mathrm{B}$ cell-targeted rituximab therapy. Additionally, there has been a general emphasis on reducing healthcare use while improving the quality of care.

We used a retrospective, serial cross-sectional design to analyze data from the nationally representative Kids' Inpatient Database (KID) to examine US trends in inpatient healthcare use and mortality associated with pediatric SLE. Specifically, we aimed to (1) characterize the national pediatric SLE inpatient cohort; (2) describe trends in the hospitalization rate, inpatient mortality rate, procedure rate, and length of stay (LOS) for pediatric SLE; (3) identify patient and hospital-specific risk factors for the outcomes of mortality and LOS; and (4) compare these outcomes for patients with and without pediatric SLE.

\section{MATERIALS AND METHODS}

Study design and setting. Children and adolescents with SLE were identified from a serial cross-sectional analysis of pediatric discharges in 2000, 2003, 2006, and 2009, using data from the KID and the Healthcare Cost and Utilization Project (HCUP) of the US Agency for Healthcare Research and Quality. HCUP is the largest collection of multiyear, all-payer, encounter-level healthcare data available in the United States. The KID contains discharge data from pediatric inpatient admissions, including patient and hospital-specific variables as well as healthcare use variables such as discharge diagnoses (up to 25 per admission), procedures performed (up to 25 per admission), and LOS. The KID, available every 3 years since 1997, is a stratified sample of all pediatric discharges for children and adolescents age less than 21 years from states that participate in HCUP. Participating states provide discharge-level data on all inpatient discharges from community nonrehabilitation hospitals (nonfederal, short-term, general, and specialty hospitals) in that state. Patient-level data is not reported; therefore, individual patients cannot be tracked. The KID 2000 includes 2784 hospitals in 27 states; KID 2003 includes 3438 hospitals in 36 states; KID 2006 includes 3739 hospitals in 38 states; KID 2009 includes 4121 hospitals in 44 states. The KID was specifically designed to report pediatric hospital use and outcomes for rare pediatric conditions, and samples $10 \%$ of uncomplicated births and $80 \%$ of all other pediatric discharges from all participating hospitals to allow adequate representation of those conditions. National estimates with $95 \%$ CI can be calculated using sampling weights, which are based on hospital characteristics. More detailed information on the KID database can be found at http://www.hcup-us.ahrq.gov/kidoverview.jsp.

The Institutional Review Board at the Children's Hospital of Philadelphia deemed our study not human subjects research because of the de-identified nature of the data.

Identification of sample. We identified hospitalizations for patients with SLE ages 2 to 21 years in the KID using an International Classification of Diseases, 9th revision, Clinical Modification (ICD-9-CM) code of 710.0 listed as a discharge diagnosis. Given the systemic nature of SLE, we included those with a diagnosis of SLE in any position to optimally reveal the broad range of reasons for hospitalization. We also identified a subgroup of patients with SLE nephritis using a combination of SLE ICD-9-CM code 710.0 and 1 of several nephritis ICD-9-CM codes as described in the literature $7,12,13,14,15$ (Appendix 1). We further characterized the SLE cohort by identifying the most common principal discharge diagnoses, using Clinical Classifications Software (CCS) codes to first identify broad categories ${ }^{16}$, then individual ICD-9-CM codes to tabulate the principal discharge diagnoses. We sought to exclude neonatal patients with SLE by restricting our analyses to hospitalizations for patients aged 2 years and older. We included patients aged 18 to 21 years because this age group frequently receives care in the pediatric setting.
Outcome variables. We calculated national estimates with $95 \% \mathrm{CI}$ and evaluated for temporal trend for the outcomes of hospitalization rate, inpatient mortality rate, procedure rate, and average LOS for 2000, 2003, 2006, and 2009. We examined patient and hospital-specific risk factors for the outcomes of mortality rate and LOS, and conducted secondary analyses in the SLE nephritis subgroup. We also compared these outcomes to those without an SLE diagnosis. The annual hospitalization rate was calculated by dividing the estimated number of pediatric SLE hospitalizations by the age-matched US population estimate for that year (obtained from US Census data), and multiplying by 100,000. Annual inpatient mortality rate was calculated by dividing the number of pediatric SLE inpatient deaths by the total number of pediatric SLE hospitalizations for that year. We tabulated the most common procedures (including primary and secondary) by using CCS codes to first identify broad categories ${ }^{16}$, then individual ICD-9-CM codes to form procedure groupings (Appendix 2). The procedure rate was defined as the number of procedures performed per 1000 hospitalizations. Data for outcome variables were missing as follows: mortality $<0.1 \%$, LOS $<0.1 \%$.

Demographic variables. We included the following patient-specific characteristics in the analysis: presence of SLE nephritis (yes/no), age, race/ethnicity, sex, and health insurance coverage. We identified patients with SLE nephritis based on ICD-9-CM codes as described. We defined 3 age groups based on the American Academy of Pediatrics developmental stages $^{17}$ : children $2-11$ years, adolescents $12-17$ years, and older adolescents 18-20 years. Self-reported race/ethnicity was categorized into the following mutually exclusive groups: white, black, Hispanic, and other (includes Asian/Pacific Islander and Native American). Health insurance coverage was categorized into 3 groups based on primary payer: Medicaid (government-provided insurance for low-income persons), private, and other [includes Medicare (government-provided senior citizens' insurance), self-pay, and no charge]. We included the following hospital-specific characteristics in the analysis: hospital type (general or children's), teaching status, hospital location (rural or urban), and hospital region (Northeast, Midwest, South, or West). The classification scheme of the National Association of Children's Hospitals and Related Institutions was used to categorize hospital type into 2 groups: children's hospital (includes children's general hospital, children's specialty hospital, and children's unit in a general hospital) and general hospital (does not have a children's unit). Hospitals were considered to be teaching hospitals if they met any of the following criteria: residency training approval by the Accreditation Council for Graduate Medical Education; membership in the Council of Teaching Hospitals; or a ratio of fulltime equivalent interns and residents to beds of 0.25 or higher. Demographic data were missing for $<10 \%$ of hospitalizations, with the exception of race/ethnicity data (16\%), which was retained in the analysis because of the known differential prevalence and severity of SLE according to race/ethnicity ${ }^{1,18,19}$.

Statistical analysis. We performed all analyses and statistical comparisons using Stata 12 (Stata Corp.). National estimates with 95\% CI were calculated using the discharge-level statistical weights provided by KID. We used a marginal model with the Stata survey command to analyze the data at the population level, accounting for multilevel clustering (e.g., hospital, region) and to incorporate the sampling weights. Patient and hospitalspecific demographic variables were tabulated for pediatric SLE discharges for 2000, 2003, 2006, and 2009. We tabulated the frequencies of the principal discharge diagnoses. Hospitalization rate was evaluated for temporal trend using univariable Poisson regression. Mortality was evaluated for temporal trend, adjusting for demographic variables, using multivariable logistic regression. To evaluate for temporal trend in procedure rate and average LOS, we used a multivariable generalized linear regression model that adjusted for demographic variables, using the $\gamma$ family of distributions with a $\log$ link. This model provides reliable estimates for highly skewed outcomes such as inpatient $\operatorname{LOS}^{20}$. We tested for differences in temporal trends of mortality and LOS for those with and without an SLE diagnosis using an interaction term between diagnosis

Personal non-commercial use only. The Journal of Rheumatology Copyright @ 2014 . All rights reserved. 
group (SLE vs non-SLE) and year. We performed secondary analyses of the outcomes for patients with SLE nephritis. Throughout the analysis, all testing was 2-sided, with a threshold for statistical significance of $\mathrm{p}<0.05$.

\section{RESULTS}

Demographics and principal discharge diagnoses. Of the about 29 million total pediatric hospitalizations in the KID for the years of study, an estimated 26,903 (95\% CI 23,802-30,005) SLE hospitalizations were identified. Of those hospitalizations, $85 \%$ were of female patients, $45 \%$ were of patients age 18-20 years, and $81 \%$ were of patients of nonwhite race/ethnicity. Medicaid was identified as the primary payer in $46 \%$. Over half the hospitalizations were for patients with SLE nephritis (57\%), and this proportion was stable over time (range 55-57\%, $\mathrm{p}=0.32$ ). Additional demographic characteristics are listed in Table 1. The most commonly listed principal discharge diagnoses were SLE (45\% with ICD-9-CM 710.0), infection (11\%), and nephritis $(4 \%)$.

Hospitalization and inpatient mortality rates. The annual hospitalization rate for pediatric SLE was stable over the years of study at an average of 8.6 per 100,000 population (95\% CI 7.6-9.6; Table 2). The average inpatient mortality rate across all years was $1 \%$ (95\% CI 0.8-1.1), and there was a statistically significant decrease over the years of study to a low of $0.6 \%$ in 2009 (OR 0.95, 95\% CI 0.900-0.998, $\mathrm{p}=0.04$; Table 2). Factors associated with death were SLE nephritis, age 18-20 years, black race, and hospital location in the South region (Table 3). For the subgroup of patients with SLE nephritis, the average inpatient mortality rate was $1.4 \%$ (95\% CI 1.1-1.6), and there was a statistically significant decrease from $1.5 \%$ in 2000 to $0.7 \%$ in 2009 (OR $0.93,95 \%$ CI $0.88-0.99, \mathrm{p}=$ 0.01 ; Figure 1). Mortality for all other KID hospitalizations also showed a statistically significant but very minor decrease over time, from $0.34 \%$ in 2000 to $0.27 \%$ in 2009 (OR 0.97, 95\% CI 0.96-0.97, p < 0.001; Figure 1).

Procedure rates. About $60 \%$ of all SLE hospitalizations $(n=$ 16,056) had at least 1 billed procedure, and the median number of procedures per hospitalization was 2 (interquartile range 1-3). The most commonly billed procedures were medication infusion $(12.8 \%)$, dialysis $(8.4 \%)$, blood transfusions $(7.5 \%)$, vascular catheterization $(6.4 \%)$, and renal biopsy $(6.3 \%$; Figure $2 \mathrm{~A})$. There was a statistically significant increase in the rates of dialysis, blood transfusion, and vascular catheterization procedures performed over the years of study (Figure 2B).

Length of stay. The mean LOS of 5.9 days (95\% CI 5.6-6.2) for SLE hospitalizations was stable over time (Table 2). Factors associated with increased LOS were SLE nephritis, other race, urban location, and teaching hospital status (Table 3). Non-Medicaid payer was associated with decreased LOS. For patients with SLE nephritis, LOS was also stable over time, at a mean of 6.7 days $(95 \% \mathrm{CI}$
Table 1. Demographics for hospitalizations with a diagnosis of SLE.

\begin{tabular}{|c|c|}
\hline $\mathrm{n}=26,903$ for All Years & $\mathrm{n}(\%)$ \\
\hline \multicolumn{2}{|l|}{ Patient-specific factors } \\
\hline \multicolumn{2}{|l|}{ Age, yrs } \\
\hline $2-12$ & $2337(8.7)$ \\
\hline $12-17$ & $12,379(46.0)$ \\
\hline $18-20$ & $12,187(45.3)$ \\
\hline Female* & $22,814(84.8)$ \\
\hline \multicolumn{2}{|l|}{ Race/ethnicity* } \\
\hline White & $5140(19.1)$ \\
\hline Black & $8853(32.9)$ \\
\hline Hispanic & $5902(21.9)$ \\
\hline Other & $2733(10.2)$ \\
\hline \multicolumn{2}{|l|}{ Primary payer* } \\
\hline Medicaid & $12,268(45.6)$ \\
\hline Private & $10,411(38.7)$ \\
\hline Other & $4171(15.5)$ \\
\hline \multicolumn{2}{|l|}{ SLE nephritis } \\
\hline No & $11,705(43.5)$ \\
\hline Yes & $15,198(56.5)$ \\
\hline \multicolumn{2}{|l|}{ Hospital-specific factors } \\
\hline \multicolumn{2}{|l|}{ Location* } \\
\hline Urban & $24,858(92.4)$ \\
\hline Rural & $1025(3.8)$ \\
\hline \multicolumn{2}{|l|}{ Region } \\
\hline Northeast & $5165(19.2)$ \\
\hline Midwest & $4246(15.8)$ \\
\hline South & $10,478(38.9)$ \\
\hline West & $7014(26.1)$ \\
\hline \multicolumn{2}{|l|}{ Teaching status* } \\
\hline Teaching & $20,608(76.6)$ \\
\hline Non-teaching & $5281(19.6)$ \\
\hline \multicolumn{2}{|l|}{ Type* } \\
\hline Children's Hospital & $14,958(55.6)$ \\
\hline General Hospital & $9990(37.1)$ \\
\hline
\end{tabular}

* The percentages for these categories do not add up to $100 \%$ because of missing demographic data, as follows: sex $0.3 \%$, race/ethnicity $15.9 \%$, primary payer $0.2 \%$, location $3.8 \%$, teaching status $3.8 \%$, hospital type 7.3\%. SLE: systemic lupus erythematosus.

6.2-7.1). LOS estimates for SLE hospitalizations were higher than the average LOS of 3.6 days (95\% CI 3.6-3.7) for those without SLE, which was also stable over time.

\section{DISCUSSION}

This retrospective serial cross-sectional analysis provides national estimates of US inpatient healthcare use trends for pediatric SLE over a decade. Using administrative data from the nationally representative KID, we identified about 27,000 US hospitalizations for pediatric SLE over a 4-year period. The characteristics of this national inpatient pediatric SLE cohort are consistent with previously reported age, race, and sex demographics of pediatric SLE populations $1,7,18,21$. It is notable that almost half the cohort was 18-20 years of age, which likely reflects the onset of pediatric SLE during adolescence. Over half of the SLE hospitalizations $(57 \%)$ were for those with SLE nephritis, which was expected given the significant morbidity and

$$
\text { Personal non-commercial use only. The Journal of Rheumatology Copyright @ } 2014 \text {. All rights reserved. }
$$


Table 2. Temporal trends in SLE hospitalization rate, mortality, and LOS.

\begin{tabular}{|c|c|c|c|c|}
\hline Year & $\begin{array}{l}\text { Discharges, } \mathrm{n} \\
(95 \% \mathrm{CI})\end{array}$ & $\begin{array}{l}\text { Hospitalization Rate* } \\
\qquad(95 \% \text { CI })\end{array}$ & $\begin{array}{l}\text { Mortality, \% } \\
\text { (95\% CI) }\end{array}$ & $\begin{array}{c}\text { Mean LOS, days } \\
(95 \% \text { CI })\end{array}$ \\
\hline All yrs & $26,903(23,802-30,005)$ & $8.6(7.6-9.6)$ & $1.0(0.8-1.1)$ & $5.9(5.6-6.2)$ \\
\hline 2003 & $6701(5844-7559)$ & $8.6(7.6-9.7)$ & $1.2(0.8-1.5)$ & $6.1(5.7-6.5)$ \\
\hline 2006 & $7344(6416-8273)$ & $9.3(8.2-10.5)$ & $1.2(0.9-1.5)$ & $6.3(5.8-6.8)$ \\
\hline 2009 & $6896(6021-7770)$ & $8.7(7.6-9.7)$ & $0.6(0.4-0.8)$ & $5.7(5.3-6.0)$ \\
\hline
\end{tabular}

* SLE hospitalizations per 100,000 children in population aged 2 to 21 years, based on population estimates from US Census data. ** Outcomes evaluated for temporal trend using multivariable regression to adjust for patient and hospital-specific demographic variables (with the exception of hospitalization rate, which was evaluated by univariable regression). SLE: systemic lupus erythematosus; LOS: length of stay.

Table 3. Risk factors for inpatient mortality and LOS for SLE hospitalizations.

\begin{tabular}{|c|c|c|}
\hline Risk Factor & $\begin{array}{c}\text { Mortality, } \\
\text { OR }(95 \% \text { CI) }\end{array}$ & $\begin{array}{c}\text { LOS, } \\
\text { RR }(95 \% \mathrm{CI})\end{array}$ \\
\hline \multicolumn{3}{|l|}{ Nephritis } \\
\hline No & - & - \\
\hline Yes & $2.71(1.74-4.23)^{* * *}$ & $1.30(1.21-1.40)^{* * *}$ \\
\hline \multicolumn{3}{|l|}{ Age, yrs } \\
\hline $2-12$ & - & - \\
\hline $12-17$ & $1.42(0.58-3.48)$ & $0.92(0.81-1.04)$ \\
\hline $18-20$ & $2.85(1.21-6.71)^{*}$ & $1.06(0.93-1.20)$ \\
\hline \multicolumn{3}{|l|}{ Sex } \\
\hline Female & - & - \\
\hline Male & $1.51(0.99-2.29)$ & $1.03(0.94-1.14)$ \\
\hline \multicolumn{3}{|l|}{ Race } \\
\hline White & - & - \\
\hline Black & $2.01(1.12-3.62)^{*}$ & $1.09(1.00-1.20)$ \\
\hline Hispanic & $1.39(0.73-2.64)$ & $1.07(0.97-1.18)$ \\
\hline Other & $1.88(0.94-3.75)$ & $1.14(1.001-1.30)^{*}$ \\
\hline \multicolumn{3}{|l|}{ Payer } \\
\hline Medicaid & - & - \\
\hline Private & $0.89(0.59-1.34)$ & $0.88(0.82-0.94)^{* * *}$ \\
\hline Other & $1.01(0.65-1.58)$ & $0.90(0.82-0.999)^{*}$ \\
\hline \multicolumn{3}{|l|}{ Location } \\
\hline Rural & - & - \\
\hline Urban & $0.98(0.28-3.42)$ & $1.33(1.16-1.53)^{* * *}$ \\
\hline \multicolumn{3}{|l|}{ Region } \\
\hline Northeast & - & - \\
\hline Midwest & $0.81(0.34-1.95)$ & $1.08(0.92-1.28)$ \\
\hline South & $2.03(1.23-3.36)^{* *}$ & $1.06(0.92-1.21)$ \\
\hline West & $1.77(0.98-3.20)$ & $1.11(0.93-1.32)$ \\
\hline \multicolumn{3}{|l|}{ Teaching } \\
\hline No & - & - \\
\hline Yes & $1.06(0.60-1.87)$ & $1.10(1.01-1.20)^{*}$ \\
\hline \multicolumn{3}{|l|}{ Type } \\
\hline General & - & - \\
\hline Children's & $0.95(0.59-1.52)$ & $1.05(0.95-1.17)$ \\
\hline
\end{tabular}

Shown are OR and relative risks (RR) for risk factors included in the multivariable regressions for the main outcomes. The covariates included in the multivariable regressions were calendar year, presence of SLE nephritis, age group, sex, race/ethnicity, primary payer, hospital location, hospital region, hospital teaching status, and hospital type. Reference groups are denoted by "-_". Significance for $\mathrm{p}$ values is $* \mathrm{p}<0.05$, ** $\mathrm{p}<0.01$, $* * * \mathrm{p}<0.001$. SLE: systemic lupus erythematosus; LOS: length of stay. mortality association with SLE renal disease, and we found that this proportion was stable over time. Our study extends the work of Tanzer, et al, who found similar demographics and a high proportion of inpatients with SLE nephritis, using KID data from 2000 to $2006^{7}$.

We present previously unreported national US data on inpatient hospitalization and mortality rates for pediatric SLE. We found an average annual rate of 8.6 hospitalizations for pediatric SLE per 100,000 population. Given the estimated prevalence of childhood-onset SLE at 3 to 9 per 100,000 population $^{1}$, this estimated hospitalization rate suggests that children with SLE will have an average of 1-3 hospitalizations every year. Although not reflective of the rate per individual, it represents a substantial burden from the healthcare system perspective. Our estimate for average all-cause mortality in patients with SLE was $1 \%$, and $1.4 \%$ for those with SLE nephritis, both higher than the rate of $0.3 \%$ for those without SLE. Encouragingly, we found that inpatient SLE deaths decreased significantly over time from $1 \%$ to $0.6 \%$, paralleled by a decrease in those with SLE nephritis and a minor decrease for those without SLE. This downward trend may represent fluctuation over time, but we think it likely represents a real decrease in mortality given the similar trend in both SLE groups. We speculate that this observed decrease in inpatient SLE mortality may be attributable to several circumstances. Pediatric care for acute SLE flares may be improving owing to better access to outpatient and inpatient rheumatology and other subspecialty care; earlier diagnosis and treatment leading to less severe flares and improved longterm disease control; increased availability of newer and less toxic immunosuppressive treatments such as MMF and rituximab therapy with less cyclophosphamide exposure ${ }^{8,9,10,11}$; and overall improved pediatric healthcare technology and intensive care.

While the above factors may be influencing the decreasing inpatient mortality rate for pediatric SLE, LOS appears to be unaffected and has not changed significantly over time. The longer LOS for pediatric SLE hospitaliza- 


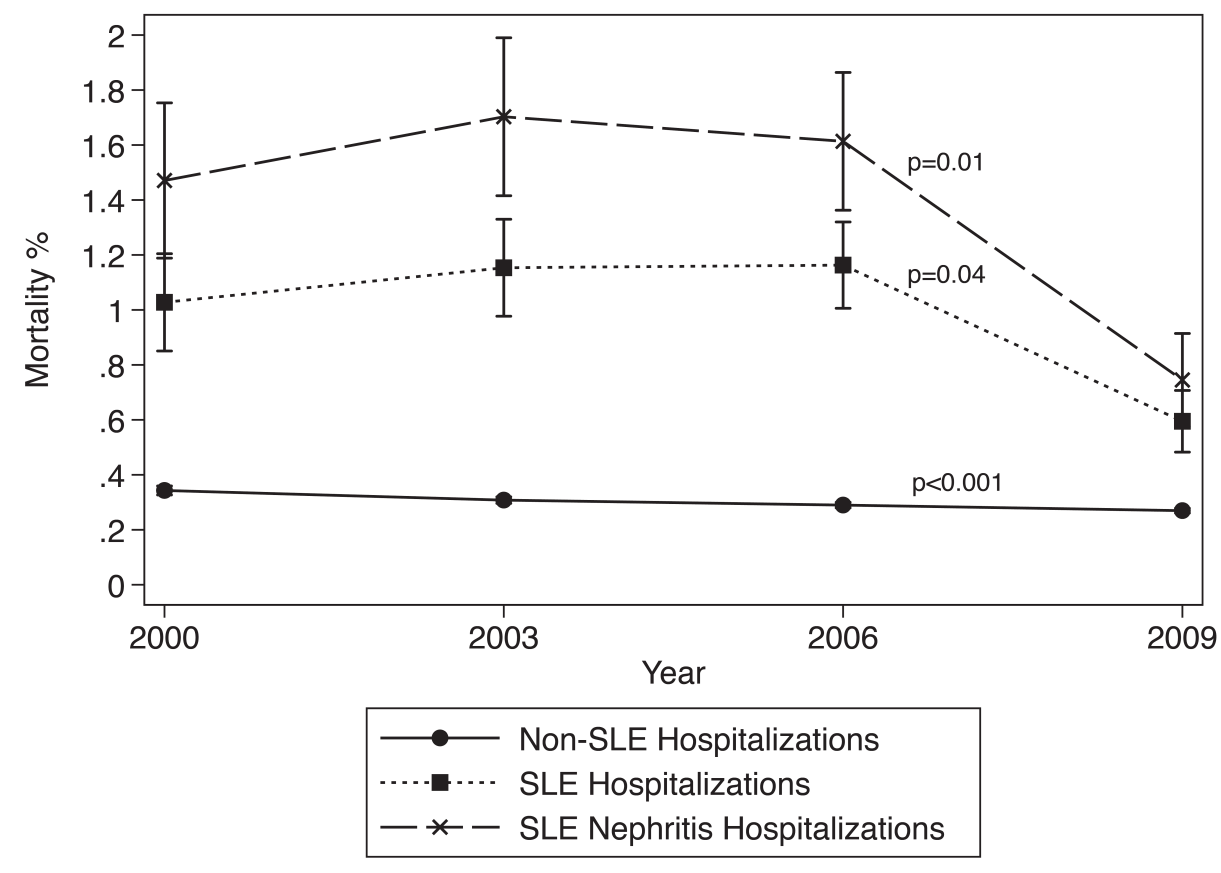

Figure 1. Inpatient mortality for children and adolescents with a diagnosis of systemic lupus erythematosus (SLE) showed a statistically significant decrease over the years of study (OR $0.95,95 \%$ CI $0.900-0.998, \mathrm{p}=$ $0.04)$. Mortality for those with SLE nephritis also showed a statistically significant decrease (OR $0.93,95 \%$ CI $0.88-0.99, \mathrm{p}=0.01)$. Mortality for all other KID hospitalizations showed a statistically significant but very minor decrease (OR $0.97,95 \%$ CI $0.96-0.97, \mathrm{p}<0.001)$. The difference in rate of mortality decrease for hospitalizations with SLE diagnosis versus those without a SLE diagnosis was not statistically significant. Error bars indicate standard error; the error bars for the group of hospitalizations without an SLE diagnosis are so small that they are barely visible on the graph. KID: US Kids' Inpatient Database.

tions at 5.9 days compared to 3.6 days for those without SLE is indicative of high healthcare use for children with SLE. Further, the longer LOS is more pronounced for those with SLE nephritis, at 6.7 days. This confirms the work of Tanzer, et al, who also reported high healthcare use for pediatric SLE, especially in those with kidney disease, in comparison to other pediatric hospitalizations ${ }^{7}$. In contrast, however, we did not find a statistically significant increase in the LOS over time. The high resource use for inpatient pediatric SLE patients is likely due to the need for more invasive procedures, multispecialty and intensive care management for SLE, and particularly SLE nephritis, in comparison to other pediatric hospitalizations. This is supported by our findings that medication infusions, dialysis, blood transfusions, vascular catheterizations, and renal biopsies were the most common procedures for SLE inpatients. We also found a statistically significant increase in the rates of dialysis, blood transfusions, and vascular catheterization procedures over the study period. Thus there appears to be an increased volume of procedures occurring over time, perhaps due to increased procedure availability and improved patient survival resulting in higher procedure rates for continued chronic care. Although the direct effect of therapeutic advances on outcomes is currently unclear, enhanced resource use may be leading to decreased inpatient mortality and improved overall survival for patients with pediatric SLE. If this trend eventually translates into decreases in pediatric SLE hospitalization rate and LOS, then the resulting overall effect may be a decrease in healthcare use. We did not observe this effect over the decade of our study, and it is possible that this effect may not be seen until later in the disease course.

We found that several patient-specific risk factors were associated with worse outcomes. Patients with SLE nephritis had increased inpatient mortality and LOS, which is consistent with expected higher morbidity and mortality in this group. Older age was associated with increased inpatient mortality, likely due to increasing complications of SLE and its treatment with age. Similarly, the increased mortality risk and LOS for patients of nonwhite race is consistent with findings of more severe SLE in nonwhite populations ${ }^{18,19}$. Medicaid patients had an increased LOS, suggesting a role for lower socioeconomic status in the risk for worse outcomes, but we did not find an association with SLE mortality, as previously reported ${ }^{22}$. For hospitalspecific factors, location in the South region was associated with increased mortality, for reasons that are unclear. Although there was a higher proportion of nonwhite and Medicaid patients in this region (data not shown) with potentially higher disease severity, location in the South

Personal non-commercial use only. The Journal of Rheumatology Copyright (c) 2014. All rights reserved. 
A.
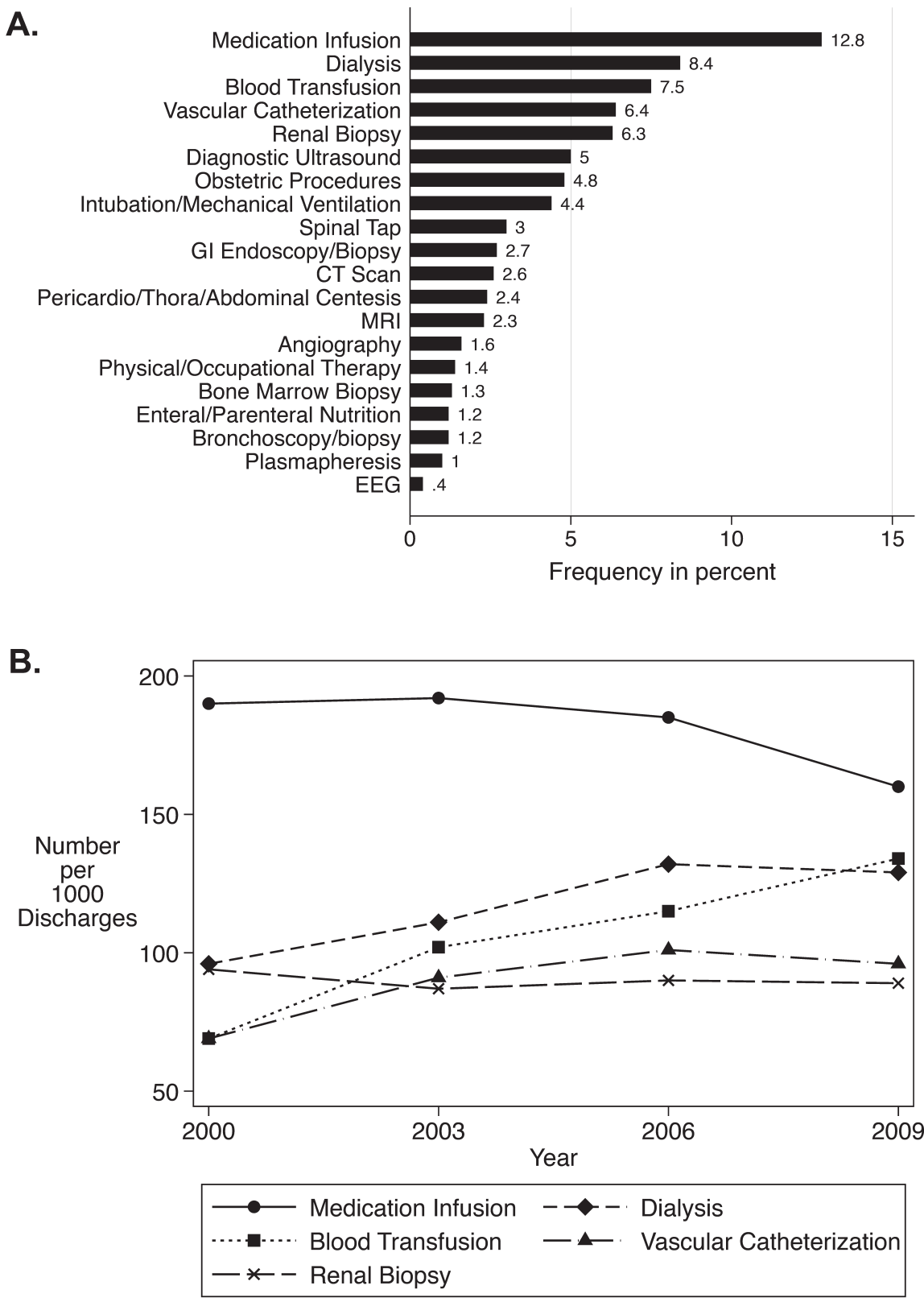

Figure 2. A. The most common $75 \%$ of billed procedures during hospitalization for systemic lupus erythematosus are shown. GI: gastrointestinal; CT: computed tomography; MRI: magnetic resonance imaging; EEG: electroencephalography. B. Temporal trends for the 5 most commonly billed hospital procedures (comprising over $40 \%$ of all procedures) are shown. The associated $\mathrm{p}$ values for trend are medication infusion $(\mathrm{p}=0.20)$, dialysis $(\mathrm{p}=$ $0.01)$, blood transfusion $(p<0.001)$, vascular catheterization $(p=0.002)$, and renal biopsy $(p=0.74)$.

region was an independent risk factor, and this may be due to other unmeasured factors related to SLE disease severity.

Our study presents the first estimates, to our knowledge, of inpatient hospitalization and mortality rates for pediatric SLE in the United States, as well as relevant healthcare use trends. The strength of our study lies in the large number of observations representative of the national pediatric SLE population. Despite this, a potential limitation is the lack of validation for the single ICD-9-CM code of 710.0 for identi- fication of SLE using administrative data, which could lead to inaccurate outcome estimates; however, the code has been validated in coding algorithms using administrative data for identification of patients with SLE nephritis ${ }^{12}$. Importantly, we identified those with nephritis as a significant proportion of the SLE inpatients with higher healthcare use and mortality rates, and it is possible that the prevalence of nephritis may actually be higher if patients were admitted for another reason, and the recorded ICD-9-CM codes did 
not reveal a history of nephritis. Unfortunately, we were unable to characterize other clinical manifestations and disease severity; the KID does not contain clinical details; and severity measures, such as the All Patient Refined Diagnosis Related Groups, were not available for all years of interest, and were not reliable because coding algorithms changed over time.

Our analysis of healthcare use trends over a decade provides valuable insight into the burden of SLE care from both the patient and healthcare system perspectives. Overall resource use by patients with SLE, and especially those with nephritis, has remained high; however, we were unable to determine individual patient burden because patients could not be tracked over time owing to the lack of unique patient identifiers in the KID, and some patients may be represented more than once in the dataset. We thus think that our estimates are especially meaningful from an overall healthcare burden perspective, and we indeed found a high average LOS for patients with SLE who are likely to have repeated hospitalizations during the course of their chronic disease, compared to those without SLE. Our finding of increasing rates of common therapeutic procedures also reflects the high overall healthcare burden for pediatric SLE. Coding practices may have affected the completeness of ICD-9-CM procedure codes, because the recorded codes may be more representative of invasive procedures (such as dialysis) than noninvasive procedures (such as magnetic resonance imaging), but our data still shed light on the frequency of invasive and often more costly procedures. Further evaluation of national healthcare costs for pediatric SLE would be a helpful additional measure of healthcare use; however, we were unable to accurately evaluate costs in the KID because of unavailability, missingness, and imprecision of cost-to-charge ratios for the years of study. Given the potential effect of MMF and other new treatment regimens, detailed analysis of the relationship between trends in specific treatments and the outcomes of interest would also be informative, but we were unable to address this because of the lack of itemized billing information in the KID.

We present novel data that raise interesting questions relevant to the patient and healthcare system burden of inpatient care as well as survival for patients with pediatric SLE. While our findings indicate that the US national hospitalization rate and LOS have remained stable for pediatric SLE, they represent a significant disease burden compared to those without SLE. The rates of common therapeutic procedures are increasing, while inpatient mortality is decreasing. Therefore, overall outcomes may be improving for patients with pediatric SLE, but certain groups remain at high risk for increased healthcare use and death. More research is needed to understand the role of advances in treatment and healthcare technology in the changing course of pediatric SLE healthcare use and survival.

\section{ACKNOWLEDGMENT}

The authors thank David D. Sherry, MD, for his critical review of the manuscript and Russell Localio, PhD, for his critical review and contribution to the biostatistical methodology used in this project.

\section{REFERENCES}

1. Kamphuis S, Silverman ED. Prevalence and burden of pediatric-onset systemic lupus erythematosus. Nat Rev Rheumatol 2010;6:538-46.

2. Zhu TY, Tam LS, Li EK. Cost-of-illness studies in systemic lupus erythematosus: a systematic review. Arthritis Care Res 2011;63:751-60.

3. Slawsky KA, Fernandes AW, Fusfeld L, Manzi S, Goss TF. A structured literature review of the direct costs of adult systemic lupus erythematosus in the US. Arthritis Care Res 2011; 63:1224-32.

4. Krishnan E. Hospitalization and mortality of patients with systemic lupus erythematosus. J Rheumatol 2006;33:1770-4.

5. Brunner HI, Sherrard TM, Klein-Gitelman MS. Cost of treatment of childhood-onset systemic lupus erythematosus. Arthritis Rheum 2006;55:184-8.

6. Karve S, Candrilli S, Kappelman MD, Tolleson-Rinehart S, Tennis $\mathrm{P}$, Andrews E. Healthcare utilization and comorbidity burden among children and young adults in the United States with systemic lupus erythematosus or inflammatory bowel disease. J Pediatr 2012;161:662-70.

7. Tanzer M, Tran C, Messer KL, Kroeker A, Herreshoff E, Wickman $\mathrm{L}$, et al. Inpatient healthcare utilization by children and adolescents with systemic lupus erythematosus and kidney involvement. Arthritis Care Res 2013;65:382-90.

8. Appel GB, Contreras G, Dooley MA, Ginzler EM, Isenberg D, Jayne D, et al. Mycophenolate mofetil versus cyclophosphamide for induction treatment of lupus nephritis. J Am Soc Nephrol 2009;20:1103-12.

9. Ginzler EM, Dooley MA, Aranow C, Kim MY, Buyon J, Merrill JT, et al. Mycophenolate mofetil or intravenous cyclophosphamide for lupus nephritis. N Engl J Med 2005;353:2219-28.

10. Grootscholten C, Ligtenberg G, Hagen EC, van den Wall Bake AW, de Glas-Vos JW, Bijl M, et al. Azathioprine/methylprednisolone versus cyclophosphamide in proliferative lupus nephritis. A randomized controlled trial. Kidney Int 2006;70:732-42.

11. Houssiau FA, Vasconcelos C, D'Cruz D, Sebastiani GD, Garrido Ed Ede R, Danieli MG, et al. Immunosuppressive therapy in lupus nephritis: the Euro-Lupus Nephritis Trial, a randomized trial of low-dose versus high-dose intravenous cyclophosphamide. Arthritis Rheum 2002;46:2121-31.

12. Chibnik LB, Massarotti EM, Costenbader KH. Identification and validation of lupus nephritis cases using administrative data. Lupus 2010;19:741-3.

13. Carls G, Li T, Panopalis P, Wang S, Mell AG, Gibson TB, et al. Direct and indirect costs to employers of patients with systemic lupus erythematosus with and without nephritis. J Occup Environ Med 2009;51:66-79.

14. Li T, Carls GS, Panopalis P, Wang S, Gibson TB, Goetzel RZ. Long-term medical costs and resource utilization in systemic lupus erythematosus and lupus nephritis: a five-year analysis of a large Medicaid population. Arthritis Rheum 2009;61:755-63.

15. Pelletier EM, Ogale S, Yu E, Brunetta P, Garg J. Economic outcomes in patients diagnosed with systemic lupus erythematosus with versus without nephritis: results from an analysis of data from a US claims database. Clin Ther 2009;31:2653-64.

16. Clinical Classifications Software (CCS) for ICD-9-CM, Healthcare Cost and Utilization Project (HCUP). Agency for Healthcare Research and Quality, Rockville, MD, 2006-2009. [Internet.

Personal non-commercial use only. The Journal of Rheumatology Copyright @ 2014 . All rights reserved. 
Accessed December 19, 2013.] Available from: www.hcup-us.ahrq.gov/toolssoftware/ccs/ccs.jsp

17. American Academy of Pediatrics. Ages \& Stages. [Internet. Accessed December 19, 2013.] Available from: www.healthychildren.org/English/ages-stages

18. Levy DM, Peschken CA, Tucker LB, Chedeville G, Huber AM, Pope JE, et al. Influence of ethnicity on childhood-onset systemic lupus erythematosus: results from a multiethnic multicenter Canadian cohort. Arthritis Care Res 2013;65:152-60.

19. Hiraki LT, Benseler SM, Tyrrell PN, Harvey E, Hebert D, Silverman ED. Ethnic differences in pediatric systemic lupus erythematosus. J Rheumatol 2009;36:2539-46.
20. Basu A, Manning WG, Mullahy J. Comparing alternative models: log vs Cox proportional hazard? Health Econ 2004;13:749-65.

21. Cassidy JT. Textbook of pediatric rheumatology, 6th ed. Philadelphia, PA: Saunders/Elsevier; 2011.

22. Alarcon GS, McGwin G Jr., Bastian HM, Roseman J, Lisse J, Fessler BJ, et al. Systemic lupus erythematosus in three ethnic groups. VII [correction of VIII]. Predictors of early mortality in the LUMINA cohort. LUMINA Study Group. Arthritis Rheum 2001;45:191-202.

APPENDIX 1. ICD-9-CM codes used to define SLE nephritis. SLE diagnosis with ICD-9-CM $=710.0$ in any position, plus one of the following codes in any position.

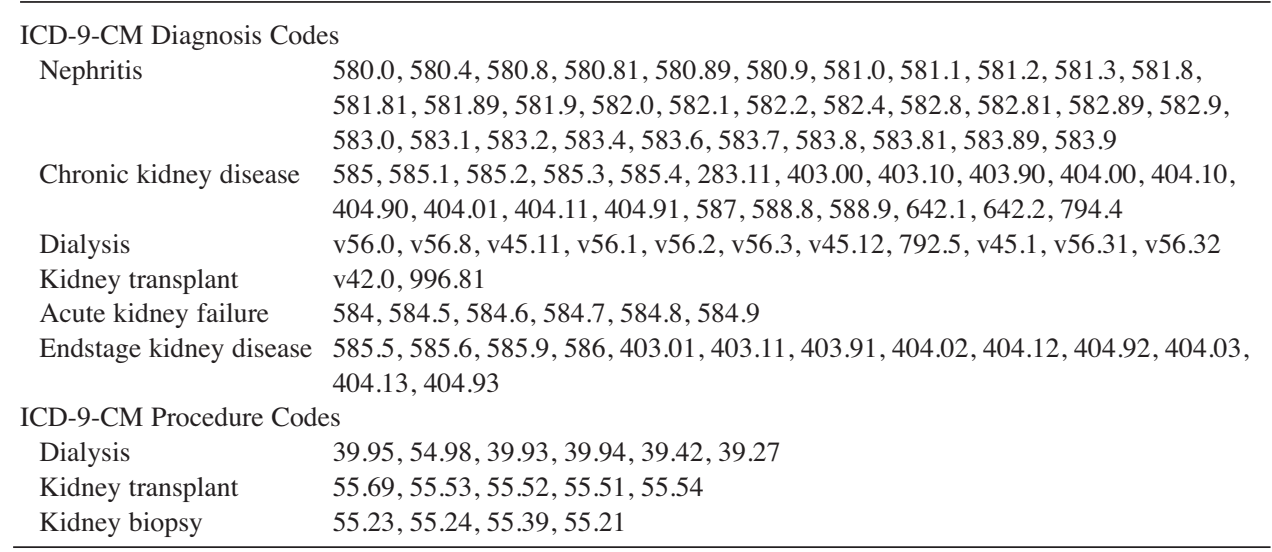

ICD-9-CM: International Classification of Diseases, 9th revision, clinical modification; SLE: systemic lupus erythematosus.

APPENDIX 2. ICD-9 code groupings for procedure categories.

\begin{tabular}{ll}
\hline Procedure Category & ICD-9-CM Procedure Codes \\
\hline Medication infusion & $9971,9929,9928,9923,9925,9921,9914,9918,9919,9910$ \\
Dialysis & $5498,3995,3994,3993,3942,3927$ \\
Blood transfusion & $9900-9909$ \\
Vascular catheterization & $3891,3892,3893$ \\
Renal biopsy & 5523,5524 \\
Diagnostic ultrasound & $8872,8873,8875,8877-8879,0023$ \\
Obstetric procedures & $740-742,7499,7301,7309,731,734,7351,7359,7531-7535$, \\
& $7538,7550-7552,7561,7562,7569$ \\
Intubation/ventilation & $9390,9604,9671,9672$ \\
Spinal tap & 331 \\
GI endoscopy/biopsy & $4223,4224,4414,4513,4514,4516,4523-4525$ \\
CT scan & $8703,8741,8801,87710031,8838$ \\
Pericardiocardial/thoracic/abdominal centesis & $3404,3409,3491,370,5491$ \\
MRI & $8891-8895,8897$ \\
Angiography & $8851,8849,8847,8843,8841,8852-8857,3721-3723$ \\
Physical/occupational therapy & $9339,9322,9311,9383,9381,9375$ \\
Bone marrow biopsy & 4131 \\
Bronchoscopy & $3322-3324,3326,3327$ \\
Enteral/parenteral nutrition & 966,9915 \\
Plasmapheresis & $9971-9974$ \\
EEG & 8914 \\
\hline
\end{tabular}

ICD-9-CM: International Classification of Diseases, 9th revision, clinical modification; GI: gastrointestinal; CT: computed tomography; MRI: magnetic resonance imaging; EEG: electroencephalography. 